\title{
Dynamic Contract Trading in Spectrum Markets
}

\author{
G.S. Kasbekar, \\ S. Sarkar, \\ K. Kar, \\ P. Muthusamy, \\ A. Gupta
}

\begin{abstract}
We address the question of optimal trading of bandwidth (service) contracts in wireless spectrum markets, for the primary spectrum providers. We propose a structured spectrum market and consider two basic types of spectrum contracts that can help attain desired flexibilities and tradeoffs in terms of service quality, spectrum usage efficiency and pricing: long-term guaranteed-bandwidth contracts, and shortterm opportunistic-access contracts. A primary provider (seller) creates and maintains a portfolio composed of an appropriate mix of these two types of contracts. The optimal contract trading question in this context amounts to how the spectrum contract portfolio of a seller in the spectrum market should be dynamically adjusted, so as to maximize return subject to meeting the bandwidth demands of its own subscribers. We formulate the optimal contract trading question as a stochastic dynamic programming problem, and obtain structural properties of the optimal dynamic trading strategy that takes into account the current market prices of the contracts and the subscriber demand process in the decision-making.
\end{abstract}

\section{INTRODUCTION}

The number of users of the wireless spectrum, as well as the demand for bandwidth per user, has been growing at an enormous pace in recent years. Since spectrum is limited, its effective management is vitally important to meet this growing demand. The spectrum available for public use can be broadly categorized into the unlicensed and licensed zones. In the unlicensed part of the spectrum, any wireless device is allowed to transmit. To use the licensed part, however, license must be obtained from appropriate government authority - the Federal Communications Commission (FCC) in the United States, for example - for the exclusive right to transmit in a certain block of the spectrum over the license time period, typically for a fee. The need for bringing market-based reform in spectrum trading, with the goal of ensuring efficient use of spectrum and fairness in allocation and pricing of bandwidth, is being increasingly recognized by both economists and engineers [4], [7], [14], [16], [15], [23]. The literature on the economics of spectrum allocation has so far mostly focused on the debate of spectrum commons [12], [14], [16] and spectrum auction mechanism design [10], [17], [22], [21]. Spectrum sharing games and/or pricing issues have been considered in [5], [6], [13], [8], [20]. A clear design of the spectrum market structure, precise definition of spectrum contracts, or how the different contracts can be optimally traded in a dynamic market environment is yet to

G.S. Kasbekar and S. Sarkar are at the Electrical and Systems Engineering Department of University of Pennsylvania, Philadelphia, PA 19104, USA. Their emails are \{kgaurav,swati\}@seas.upenn.edu. K. Kar, P. Muthusamy and A. Gupta are at Rensselaer Polytechnic Institute Troy, NY 12180, USA. Their emails are \{muthup,kark,guptaa\}@rpi.edu. emerge. This is the space in which we contribute in this paper.

We consider a spectrum market where the license holders (referred to as primary providers henceforth) can potentially sell to the secondary providers ${ }^{1}$ the spectrum they have licensed from the FCC but do not envision using in near future. Primary providers may either be providers of TV broadcasts, or large providers of wireless service who operate nationwide. Secondary providers are relatively smaller, but larger in number, and can be geographically limited providers, whose access to spectrum occurs through the bandwidth (service) contracts that they buy from primary providers. Providers in both categories have their subscriber (TV or mobile communication subscriber) bases whom they need to serve using the spectrum they respectively license from the FCC or buy in the spectrum market. This spectrum market structure is motivated by, and closely resembles, secondary financial markets used for trading of financial instruments (such as stocks, bonds) among investment banks, hedge-funds etc. Like in secondary financial markets, we allow trading in spectrum markets, not only of the raw spectrum (bandwidth), but also of the different kinds of service contracts derived from the use of spectrum. A question that is key to the efficient operation of the spectrum market is how the primary providers should trade spectrum (bandwidth/service) contracts dynamically, based on timevarying demand patterns arising from their subscribers, to maximize their returns while satisfying their subscriber base. This is the central focus of this paper.

We formulate and evaluate the solutions for the spectrum contract trading problem for the primary providers. We consider two basic forms of contracts that are used for selling/buying spectral resources: i) Guaranteed-bandwidth (Type-G) contracts, and (ii) Opportunistic-access (Type-O) contracts. Under the Type- $G$ contracts, a secondary provider purchases a guaranteed amount of bandwidth (in units of frequency bands or sub-bands) for a specified duration of time (typically a "long term") from a primary provider, and pays a fixed fee (either as a lump-sum or as a periodic payment through the duration of the contract) irrespective of how much it uses this bandwidth. If after selling the contract, the primary is unable to provide the promised bandwidth (this may for example happen when the primary is forced to use a band it has sold due to an unexpected rise in its subscriber demand), the primary financially compensates the secondary for contractual violation. On the other hand, Type-

\footnotetext{
${ }^{1}$ Note that our notion of "primary" and "secondary" spectrum providers must be distinguished from similar terms often associated with users (subscribers in our case) in the spectrum allocation literature.
} 
$O$ contracts are short-term (one time unit in our model), and a secondary which buys a Type- $O$ contract pays only for the amount of bandwidth it actually uses on the corresponding band. The primary does not provide any guarantee on a Type- $O$ contract and may use the channel sold as a Type$O$ contract without incurring any penalty. Thus, a Type- $O$ contract provides the secondary the right to use the channel if the primary is not using it.

The spectrum contract trading problem that we formulate and solve allows the primary provider to dynamically adjust its spectrum contract portfolio, i.e, choose how much of each type of contract to sell at any time, so as to maximize its profit subject to satisfying its own subscriber demand that varies with time, and given the current market prices of Type- $G$ and Type- $O$ contracts which also vary with time. We address the Primary's Spectrum Contract Trading (Primary$S C T$ ) problem in Sections II and III. We formulate the problem as a finite horizon stochastic dynamic program whose computation time is polynomial in the input size. We prove several structural properties of the optimum solutions. For example, we show that the optimal number of Type- $G$ contracts are monotone (increasing or decreasing) functions of the subscribers' demands and the contract prices. These structural results provide more insight into the problems, and allow us to develop faster algorithms for solving the dynamic programs.

Although the spectrum contract trading problem has been motivated by analogues in financial markets, the actual questions posed and the techniques used to answer them turn out to be quite different owing to the nature of the specific commodity, that is RF spectrum, under consideration. First, the primary must decide its trading strategy considering its subscriber demand which changes with time. For example, a primary can not simply decide to sell a large number of Type- $G$ contracts at any given time at which their market prices are high. This is because the primary will need to pay a hefty penalty if it can not deliver the promised bandwidth owing to an increase in its subscriber demand. The portfolio optimization literature in finance does not usually address the demand satisfaction constraint. Next, spectrum usage must satisfy certain temporal and spatial constraints that are perhaps unique. Specifically, a frequency band can not be simultaneously successfully used at neighboring locations (without causing significant interference), but can be simultaneously successfully used at geographically disparate locations. Thus, the spectrum trading solution for the primary provider must also take into account spatial constraints for spectrum reuse, and therefore the computation of the optimal trading strategy requires a joint optimization across all locations. We prove a surprising separation theorem in this context: when the same signal is broadcast at all locations, the Primary-SCT problem can be solved separately for each location and the individual optimal solutions can subsequently be combined so as to optimally satisfy the global reuse constraints, and obtain the same revenue as the solution of a computationally prohibitive joint optimization across locations (Section III).

\section{THE PRIMARY-SCT PROBLEM}

In this section, we pose and address Primary-SCT, the spectrum contract trading question from a primary provider's perspective. We formulate the problem when a primary provider owns channels in a single region (Section II-A), solve it using a stochastic dynamic program (Section II-B), and identify the structural properties of the optimal solution (Section II-C). In Section III, we formulate and solve the trading problem when the primary owns channels in multiple locations, considering the spatial reuse of channels across different locations.

\section{A. SCT in a single region}

We now define the Primary-SCT problem for a primary provider that owns $M$ frequency bands (channels) in a single region, which it sells as Type- $G$ or Type- $O$ contracts to secondary providers. We assume that each channel corresponds to one unit of bandwidth and at most one contract - either Type- $G$ or Type- $O$ - can stand leased on a channel at any time. We also assume that the market has infinite liquidity: there is a large number of buyers, and hence the primary provider can sell any or all of the channels it owns anytime and in any combination of Type- $G$ and Type- $O$ contracts.

We assume that time is slotted; at the beginning of each slot $t$, the primary determines the number of channels $x_{G}(t)$ and $x_{O}(t)$ to be sold as Type- $G$ and Type- $O$ contracts respectively. We consider optimization over a finite time horizon of $T$ time slots. A Type- $G$ ("long term") contract that is sold at the beginning of any slot $t=1, \ldots, T$ lasts till the end of the horizon. $T$ therefore represents the maximum duration of a Type- $G$ contract. Type- $O$ contracts last for a single slot from the time they are negotiated.

The prices of both types of contracts (i.e, the prices at which they can be bought/sold in the spectrum market) vary randomly with time and are determined "by the market", possibly depending on the current supply-demand balance in the market and other factors. The "per-slot" market prices for Type- $G$ and Type- $O$ contracts at time $t$ are denoted by $c_{G}(t)$ and $c_{O}(t)$ respectively. When a Type- $G$ contract is sold at slot $t$, it remains active for $T-t+1$ slots (that is, until the end of the optimization horizon), and therefore fetches a revenue of $\alpha(T-t+1) c_{G}(t)$, where $\alpha(n)$ is a (deterministic) increasing function of $n$ and captures the increase in value of a Type- $G$ contract with the number of slots for which it remains active, e.g., $\alpha(n)=n$. We assume that the process $\left\{c_{G}(t)\right\}$ (respectively, $\left\{c_{O}(t)\right\}$ ) constitutes a Discrete time Markov chain (DTMC) with a finite number of states and transition probability $H_{c, d}^{G}$ (respectively, $H_{c, d}^{O}$ ) from state $c$ to $d$. For simplicity, we assume that the DTMCs $\left\{c_{G}(t)\right\}$ and $\left\{c_{O}(t)\right\}$ are independent of each other, although our results readily extend to the case when the joint process $\left\{c_{G}(t), c_{O}(t)\right\}$ is a DTMC.

Each primary provider is associated with a randomly timevarying demand process, $\{i(t)\}$ which corresponds to its subscriber demand (of TV channel subscribers or wireless service subscribers, for example) that it must satisfy. We assume that the process $\{i(t)\}$ constitutes a DTMC with a 
finite number of states and transition probability $Q_{i j}$ from state $i$ to $j$, which is independent of the price process; each demand state corresponds to an integral amount of bandwidth consumption in subscriber demand.

We assume that the transition probabilities $\left\{H_{c, d}^{G}\right\},\left\{H_{c, d}^{O}\right\}$ and $\left\{Q_{i j}\right\}$ are known to the primary provider. They can be estimated from the history of the price and demand processes.

The contract trading is done at the beginning of time slot $t$, and $\left(x_{G}(t), x_{O}(t)\right)$ are determined after the market prices $c_{G}(t), c_{O}(t)$ and demand levels $i(t)$ are known. Let $\left(a_{G}(t), x_{O}(t)\right)$ denote the spectrum contract portfolio held by the primary during time slot $t$, i.e. the number of Type$G$ and Type- $O$ contracts that stand leased. Since Type- $G$ contracts last till the end of the time horizon, we have:

$$
a_{G}(t)=\sum_{t^{\prime} \leq t} x_{G}\left(t^{\prime}\right)
$$

The bandwidth not leased as Type- $G$ contracts or used to satisfy the demand is sold as Type- $O$ contracts. Thus, at any time $t$ :

$x_{O}(t)=K\left(a_{G}(t), i(t)\right):=\max \left\{0, M-a_{G}(t)-i(t)\right\}$.

However, for all slots, $t$, for which $a_{G}(t)+i(t)>M$, the primary will have to use channels already sold under Type- $G$ contracts to satisfy its subscriber demand, due to unavailability of additional bandwidth. In this case, the primary incurs a penalty, $Y\left(a_{G}(t), i(t)\right)$, for breaching Type$G$ contracts. The penalty is proportional to the number of such channels the provider uses for satisfying its subscriber demand. Thus,

$$
Y\left(a_{G}(t), i(t)\right)=\beta \max \left\{0, a_{G}(t)+i(t)-M\right\},
$$

where $\beta$ is the proportionality constant. We make the natural assumption that the penalty is hefty; in particular, $\beta$ is greater than or equal to the maximum possible price of a Type- $O$ contract.

The Primary-SCT problem then is to choose the primary's trading strategy $\left(\left(x_{G}(t), x_{O}(t)\right), t=1, \ldots T\right)$, so as to maximize its expected revenue, expressed as

$$
\begin{array}{r}
\boldsymbol{E}\left(\sum _ { t = 1 } ^ { T } \left(\alpha(T-t+1) c_{G}(t) x_{G}(t)+c_{O}(t) x_{O}(t)\right.\right. \\
\left.\left.-Y\left(a_{G}(t), i(t)\right)\right)\right),
\end{array}
$$

subject to relations (1)-(3). The optimum strategy must be causal in that for each $t \in\{1, \ldots T\},\left(x_{G}(t), x_{O}(t)\right)$ must be chosen by time $t$. Note that at time $t,\left\{i\left(t^{\prime}\right), c_{G}\left(t^{\prime}\right), c_{O}\left(t^{\prime}\right)\right.$ : $\left.t^{\prime}=1, \ldots, t\right\}$ are known, but $\left\{i\left(t^{\prime}\right), c_{G}\left(t^{\prime}\right), c_{O}\left(t^{\prime}\right): t^{\prime}=\right.$ $t+1, \ldots, T\}$ are not known to the primary provider. From (1) and (2), $x_{O}(t)$ is a function of $\left\{x_{G}\left(t^{\prime}\right): t^{\prime}=1, \ldots, t\right\}$ and the current demand $i(t)$. Therefore, the Primary-SCT problem as defined above reduces to finding the optimal $\left(x_{G}(t), t=1, \ldots, T\right)$.

Note that the revenue function in (4) ignores any revenue earned from the primary's subscribers. Since the subscriber demand process $i(t)$ is unaffected by the trading decisions, such revenue adds a constant offset to the revenue in (4), and therefore does not influence the optimal spectrum trading decisions.

\section{Generalizations:}

1) For a Type- $O$ contract, the secondary provider pays the primary only for the amount of bandwidth it uses. Thus, the expected revenue earned by a primary on selling such a contract equals the secondary's expected usage of such a channel times the market price of such a contract. We can incorporate this by considering the revenue from a Type- $O$ contract in slot $t$ as $\kappa c_{O}(t)$, where $\kappa$ is the secondary's expected usage of such a channel. The formulation and the results extend to this case.

2) Our formulation and results can be extended to consider the case that $i(t)$ is only an estimate of the demand in slot $t$, and the estimation error in each slot is an independent, identically distributed random variable whose distribution is known to the primary. Then, $x_{O}(t)$ must be selected so that $M-x_{O}(t)-a_{G}(t)$ is greater than or equal to the actual demand with a desired probability. Thus, $x_{O}(t)$ will be a function, $K\left(a_{G}(t), i(t)\right)$, of $\left(a_{G}(t), i(t)\right)$, which may be different from that in (2), but can nevertheless be determined from the knowledge of the distribution of the estimation error. Also, in this case, the lack of exact knowledge of the demand will force the primary to use part or whole of the bandwidth it has sold as Type- $O$ contracts to satisfy its demand. This will not incur any penalty for the primary owing to the nature of the contract, but will reduce the secondary's expected usage $\kappa$ of each channel sold as a Type- $O$ contract, and thereby reduce the expected amount $\kappa c_{O}(t)$ the secondary pays the primary for each such channel.

3) For clarity of exposition, we assumed integral demands $i(t)$. However, in practice, the demands may be fractional. For example, when a set of subscribers intermittently access the Internet on a channel, a fraction of the bandwidth on a channel is used every slot. In this case, a Type- $G$ or Type- $O$ contract may be sold on the channel (while incurring a penalty proportional to the fraction used on the channel for the former). All our results apply without change in this case.

\section{B. Polynomial-time optimal trading}

We show that the Primary-SCT problem defined in Section II-A can be solved as a stochastic dynamic program (SDP) [18]. A policy [18] is a rule, which specifies the decisions $\left(x_{G}(t)\right.$ and $\left.x_{O}(t)\right)$ at each slot $t$, as a function of the demands and prices and past decisions. Now, since the demand and prices are Markovian, the statistics of the future evolution of the system from slot $t$ onwards are completely determined by the vector $\left(a_{G}(t-1), i(t), c_{G}(t), c_{O}(t)\right)$, which we call the state at slot $t$, and the primary's decisions $\left\{x_{G}\left(t^{\prime}\right): t^{\prime}=t, \ldots, T\right\}$ under the policy being used. Now, in general, a policy may determine $\left(x_{G}(t), x_{O}(t)\right)$ at slot $t$ based on all past states and actions. However, a well-known result (Theorem 4.4.2 in [18]) shows that there exists an optimal policy which specifies the optimal $x_{G}(t)$ at any slot 
$t$ only as a (deterministic) function of the current state and $t^{2}$. We next compute such an optimal policy by solving a SDP.

For a given $t$, let $n=T-t+1$ be the number of slots remaining until the end of the horizon, and $V_{n}\left(a, i, c_{G}, c_{O}\right)$ denote the maximum possible revenue from the remaining $n$ slots, under any policy, when the current state is $\left(a_{G}(t-\right.$ $\left.1), i(t), c_{G}(t), c_{O}(t)\right)=\left(a, i, c_{G}, c_{O}\right)$. In particular, note that $V_{T}\left(0, i, c_{G}, c_{O}\right)$ is the maximum possible value of the expected revenue in (4) under any policy when $i(1)=i$, $c_{G}(1)=c_{G}$ and $c_{O}(1)=c_{O}$. The function $V_{n}($.$) is called$ the value function [18]. We have:

$$
V_{n}\left(a, i, c_{G}, c_{O}\right)=\max _{0 \leq x \leq M-a} W_{n}\left(a, i, c_{G}, c_{O}, x\right),
$$

where $W_{n}\left(a, i, c_{G}, c_{O}, x\right)=\alpha(n) c_{G} x+J\left(x+a, i, c_{O}\right)+$ $\sum_{d_{G}} \sum_{d_{O}} H_{c_{G} d_{G}}^{G} H_{c_{O} d_{O}}^{O} \sum_{j} Q_{i j} V_{n-1}\left(a+x, j, d_{G}, d_{O}\right)$, and $J\left(a_{G}(t), i(t), c_{O}(t)\right)=c_{O}(t) K\left(a_{G}(t), i(t)\right)-Y\left(a_{G}(t), i(t)\right)$,

and the maximum in (5) is over integer values of $x$ in $[0, M-a]$. Equation (5) is called Bellman's optimality equation [18] and holds because, by definition of $V_{n-1}($.$) ,$ $W_{n}\left(a, i, c_{G}, c_{O}, x\right)$ defined by (6) is the maximum possible expected revenue when $n$ slots remain until the end of the horizon and $x_{G}(t)=x$ is chosen. Note that the first two terms in (6) account for the revenue earned in slot $t$ from the sale of Type- $G$ and Type- $O$ contracts minus the penalty paid. The last term in (6) is the maximum expected revenue from slot $t+1$ onwards. We get (5) by taking the maximum over all permissible values of $x$. Denote the (largest) $x$ that maximizes $W_{n}\left(a, i, c_{G}, c_{O}, x\right)$ by $x_{n}^{*}\left(a, i, c_{G}, c_{O}\right)$. The function $x_{n}^{*}($.$) provides the optimal solution to the Primary-$ SCT problem.

Now, the value function and optimal policy can be found from (5) using backward induction [18], which proceeds as follows. Note that $V_{0}()=$.0 . Thus, $W_{1}($.$) can be$ computed using (6), and $V_{1}($.$) using (5), and similarly,$ $W_{2}(),. V_{2}(.) \ldots W_{n}(),. V_{n}($.$) can be successively computed.$ This backward induction consumes $O\left(\left(N_{G} N_{O} M^{2}\right)^{2} T\right)$ time, where $N_{G}\left(N_{O}\right)$ is the number of states in the Markov Chain $\left\{c_{G}(t)\right\}\left(\left\{c_{O}(t)\right\}\right)$ - the computation time is therefore polynomial in the input size.

Remark 1: Note that we consider a finite horizon formulation. An alternative would be to consider an infinite horizon formulation, in which a Type- $G$ contract is valid for $T$ slots from the time of sale (instead of until the end of horizon). But in this case, at a given slot, the state would include $\left(y_{1}^{G}(t), \ldots, y_{T}^{G}(t)\right)$, where $y_{j}^{G}(t)$ is the number of Type- $G$ contracts that are valid for $j$ slots more. Thus, the size of the state space is $O\left(M^{T}\right)$, which is exponential in $T$. Hence, we do not consider an infinite horizon formulation.

\footnotetext{
${ }^{2}$ Such a policy is called a deterministic Markov policy [18].
}

\section{Properties of the optimal solution}

We analytically prove a number of structural properties of the optimal policy, which provide insight into the nature of the optimal solution. Our results are quite general in that they hold not only for the $K(),. Y($.$) functions defined in$ (2), (3), but also for any functions that satisfy the following properties (which are of course satisfied by those in (2), (3)). This loose requirement allows our results to extend to the generalizations described at the end of Section II-A.

Property 1: $K(a, i)$ decreases in $a$ and $Y(a, i)$ increases in $a$ for each $i$. Hence, by (7), for each $i$ and $c_{O}, J\left(a, i, c_{O}\right)$ decreases in $a$.

Property 2: The $K(),. Y($.$) functions should be such that$ $J\left(a, i, c_{O}\right)$ is concave ${ }^{3}$ in $a$ for fixed $i, c_{O}$.

Property 3: The $K(),. Y($.$) functions should be such that,$ for each $a, J\left(a, i, c_{O}\right)-J\left(a+1, i, c_{O}\right)$ is an increasing function of $i$.

(6) We next state a technical assumption on the statistics of the demand and price processes that we need for our proofs.

Assumption 1: If $X_{i}$ is the demand in the next slot given that the present demand is $i$, or, if $X_{i}$ is the price of a Type$G$ (respectively, Type- $O$ ) contract in the next slot given that the present price is $i$, then for $i \leq i^{\prime}, X_{i} \leq_{s t} X_{i^{\prime}}\left(X_{i}\right.$ is stochastically smaller [19] than $\left.X_{i^{\prime}}\right)$, i.e., for each $b \in R$, $\operatorname{Pr}\left(X_{i}>b\right) \leq \operatorname{Pr}\left(X_{i^{\prime}}>b\right)$.

Intuitively, this assumption says that the primary's demand and the prices do not fluctuate very rapidly, and the demand (or price) in the next slot is more likely to be high when the current demand (or price) is high as opposed to when the current demand (or price) is low.

We are now ready to state the structural properties of the optimum trading policy. We defer the proofs of these properties to the Appendix.

The first property identifies the relation between $x_{n}^{*}\left(a, i, c_{G}, c_{O}\right)$ and $a$ :

Theorem 1: For each $n, i, c_{G}, c_{O}$,

$$
x_{n}^{*}\left(a+1, i, c_{G}, c_{O}\right)=\max \left(x_{n}^{*}\left(a, i, c_{G}, c_{O}\right)-1,0\right) .
$$

Intuitively, this theorem suggests that for each $n, i, c_{G}, c_{O}$, there exists an optimal portfolio level of Type- $G$ contracts, $a_{G}^{*}(t)$, such that if $a_{G}(t-1)=a$, then $x_{G}(t)$ should be chosen so as to make $a_{G}(t)=a_{G}^{*}(t)$. That is, the optimal $x_{G}(t)=a_{G}^{*}(t)-a$ (if the latter is non-negative).

Also, due to Theorem 1 , for each $n, i, c_{G}$ and $c_{O}$, it is sufficient to find $x_{n}^{*}\left(a, i, c_{G}, c_{O}\right)$ only for $a=0$ while performing backward induction, and $x_{n}^{*}\left(a, i, c_{G}, c_{O}\right)$ for other $a$ can be deduced from (8). This reduces the overall computation time by a factor of $M$ : the optimal policy can now be computed in $O\left(\left(N_{G} N_{O}\right)^{2} M^{3} T\right)$ time.

The next two results identify the nature of the dependence between $x_{n}^{*}\left(a, i, c_{G}, c_{O}\right)$ and the demand $i$ and prices $c_{G}, c_{O}$.

Theorem 2: For each $n, a, c_{G}$ and $c_{O}, x_{n}^{*}\left(a, i, c_{G}, c_{O}\right)$ is monotone decreasing in $i$.

\footnotetext{
${ }^{3}$ A function $f(k)$ with domain being a subset of the integers is concave [3] if $f(k+2)-f(k+1) \leq f(k+1)-f(k)$ for all $k$ [19]. If the inequality is reversed, $f($.$) is convex.$
} 
Theorem 2 confirms the intuition that when the primary's demand is high, it should sell fewer Type- $G$ contracts so as to reserve bandwidth to meet its demand and vice versa. At the same time, note that this result is not obvious- when the demand is lower, more free bandwidth is available, which can be sold as Type- $G$ or as Type- $O$ contracts. Clearly, the number of Type- $G$ versus Type- $O$ contracts sold would influence the states reached in the future and the revenue earned. Theorem 2 asserts that the primary should sell at least as many Type- $G$ contracts as before (that is, as for the high demand state), while possibly also increasing the number of Type- $O$ contracts to sell.

Theorem 3: $x_{n}^{*}\left(a, i, c_{G}, c_{O}\right)$ is monotone increasing in $c_{G}$ for fixed $n, a, i, c_{O}$ and monotone decreasing in $c_{O}$ for fixed $n, a, i, c_{G}$.

Theorem 3 confirms the intuition that the primary should preferentially sell the type of contract ( $G$ or $O$ ) with a "high" price.

Remark 2: Theorems 2 and 3 can be used to speed up the computation of the optimal policy using the monotone backward induction algorithm [18]. Similarly, in Theorem 7 (in the Appendix), we prove that the value function is concave, which can be used to speed up the computation of $x_{n}^{*}($.$) from the value function since the maximizer in$ (5) can be found in $O(\log M)$ time using a binary search like algorithm [9]. In both cases, the worst case asymptotic running time remains the same, although substantial savings in computation can be obtained in practice.

\section{SCT ACROSS MULTIPLE LOCATIONS}

We now consider spectrum contract trading across multiple locations from a primary provider's point of view. Wireless transmissions suffer from the fundamental limitation that the same channel can not be successfully used for simultaneous transmissions at neighboring locations, but can support simultaneous transmissions at geographically disparate locations. Thus, a primary provider can not trade contracts in the same channel at neighboring locations, but can do so at far off locations. Hence, the spectrum contract trading problem at different locations is inherently coupled, and must be optimized jointly. We now extend the problem formulation to consider the case of multiple locations, taking into account possible interference relationships between adjacent regions.

Consider an undirected graph $\mathcal{G}$ with the set of nodes $S$. Each node represents a certain area at some location in the overall region under consideration. There is an edge between two nodes if and only if transmissions at the corresponding locations on the same channel interfere with each other. A primary provider can sell contracts on $M$ channels at each location. At any time slot, at a given node, on each channel (a) either a Type- $G$ contract can be sold, (b) a Type- $O$ contract can be sold or (c) no contract can be sold, subject to the constraint that at no point in time, a contract can stand leased at neighbors on the channel, that is, the set of nodes at which a contract stands leased constitutes an independent set [24].
A primary provider needs to satisfy its subscriber demand which is also subject to certain reuse constraints. We consider the case where the subscribers of a primary provider require broadcast transmissions. This, for example, happens when the primary is a TV transmitter which broadcasts signals across all regions over different channels. At any given slot $t$, the primary needs to broadcast over a certain number, say $i(t)$, channels which randomly varies with time depending on subscriber demands. Whenever the primary broadcasts on a channel, the broadcast reaches all nodes, and thus the channel can not be used by the secondaries at any node. Hence, if the primary has sold a Type- $G$ contract on the channel at any node it incurs a penalty of $\beta$ at the node. Thus, at slot $t, i(t)$ represents the primary's demand at all nodes. Note that the set of nodes at which the primary uses a given channel for demand satisfaction does not constitute an independent set (as opposed to the set of nodes at which contracts stand leased). Also, the primary's usage status on any given channel at any given time (i.e., whether or not the primary is using the channel for subscriber demand satisfaction) is the same across all nodes.

The durations of Type- $G$ and Type- $O$ contracts are as described in Section II-A. We assume that at any slot $t$, Type- $G$ (respectively, Type- $O$ ) contracts have equal prices $c_{G}(t)$ (respectively, $c_{O}(t)$ ) at all nodes. The processes $\left(i(t), c_{G}(t), c_{O}(t)\right)$ evolve as per independent DTMCs as stated in Section II-A.

The spectrum contract trading problem across multiple locations for a primary (Primary-SCTM) is to optimally choose at each slot $t$, the type of contract to sell (if any) at each location on each channel so as to maximize the total expected revenue from all nodes over a finite horizon of $T$ slots.

Theorem 4: Primary-SCTM is NP-Hard.

Proof: See the Appendix.

We now characterize the optimal solution of the PrimarySCTM problem.

Lemma 1: Consider the class of policies $\mathcal{F}$, such that a policy $f \in \mathcal{F}$ operates as follows. At the beginning of the horizon, it finds a maximum independent set, $I(S)$, in $\mathcal{G}$. Then, in each slot, it sells contracts only at nodes in $I(S)$. There exists a policy in $\mathcal{F}$ that optimally solves the PrimarySCTM problem.

Proof: See the Appendix.

We refer to a policy in $\mathcal{F}$, which at each node in $I(S)$, sells contracts according to the optimal solution of the Primary-SCT problem with demand and price processes $\left\{i(t), c_{G}(t), c_{O}(t)\right\}$ as a Separation Policy.

Theorem 5 (Separation Theorem): The Separation Policy optimally solves the Primary-SCTM problem.

Proof: By Lemma 1, we can restrict our search for an optimal policy to the policies in $\mathcal{F}$. Now, the total revenue of a policy in $\mathcal{F}$ is the sum of the revenues at the nodes in $I(S)$. Clearly, the total revenue is maximized if the stochastic dynamic program for the single node case is executed at each node. Note that this solution satisfies the spatial reuse constraints since $I(S)$ is an independent set. 
Note that the optimum solution at any node can be computed in polynomial time using the SDP presented in Section II-A. However, computation of a maximum size independent set is an NP-hard problem [11]. This computation therefore seems to be the basis of the NP-hardness of Primary-SCTM. Also, the following theorem, which is a direct consequence of Theorem 5, shows that PrimarySCTM can be approximated in polynomial time within a factor of $\mu$ if the maximum independent set problem can be approximated in polynomial time within a factor of $\mu$.

Theorem 6 (Approximate Separation Theorem): Consider a $\mu$-separation policy that differs from a separation policy in that it sells contracts as per the single node optimum solution, at each node of an independent set whose size is at least $\frac{1}{\mu}$ times that of a maximum independent set. This policy's expected revenue is at least $\frac{1}{\mu}$ times the optimal expected revenue.

However, in a graph with $N$ nodes, the maximum size independent set problem can not in general be approximated to within a factor of $O\left(N^{\epsilon}\right)$ for some $\epsilon>0$ in polynomial time unless $P=N P$ [1]. Nevertheless, polynomial time approximation algorithms (PTAS) i.e., algorithms that compute an independent set whose size is within $(1-\epsilon)$ of the maximum size independent set, for any given $\epsilon>0$, using a computation time of $O\left(N^{1 / \epsilon}\right)$ are known in important special cases, e.g., when the degree of each node is upperbounded [2] (this happens in our case when the number of locations each location interferes with is upper-bounded). Thus, in view of Theorem 6 , for any given $\epsilon>0$, the Primary-SCTM problem can be approximated within a factor of $1-\epsilon$ using a computation time of $O\left(N^{1 / \epsilon}\right)$ in such graphs.

\section{CONCLUSION}

In this paper, we proposed a spectrum market with two types of contracts and studied the problem of selecting an optimal portfolio of these two contract types for the primary providers. We formulated the problem using stochastic dynamic programming and analytically proved a number of structural properties of the optimal solutions. We believe that the insights provided by our study will help in the design of future spectrum markets. A direction for future research is the analysis of the contract selection problem for the secondary providers.

\section{ACKNOWLEDGMENTS}

The contributions of G.S. Kasbekar and S. Sarkar have been supported by NSF grants 0621782, 0721308, 0914955, 0915203, 0915697 and those of K. Kar, P. Muthusamy and A. Gupta by NSF grant 0916958.

\section{REFERENCES}

[1] S. Arora, C. Lund, R. Motwani, M. Sudan, M. Szegedy "Proof Verification and Hardness of Approximation Problems", in Proc. of FOCS 1992, pp. 14-23, Oct. 1992

[2] P. Berman, M. Furer, "Approximating Maximum Independent Set in Bounded Degree Graphs", in Proc. of Symp. on Discrete Algorithms, pp. 365 - 371, 1994

[3] S. Boyd, L. Vandenberghe, Convex Optimization, Cambridge University Press, 2004.
[4] C.E Caicedo and M.B.H Weiss, "Spectrum trading: An analysis of implementation issues", in Proceedings of DySPAN 2007, pages 579584, 2007.

[5] A.A. Daoud, M. Alanyali, and D. Starobinski, "Secondary pricing of spectrum in cellular cdma networks", in Proceedings of DySPAN 2007, pages 535-542, 2007.

[6] R Etkin, A Parekh, and D Tse, "Spectrum sharing for unlicensed bands", IEEE Journal on Selected Areas in Communications, 25(3):517528, Apr 2007.

[7] G. R. Faulhaber, "The question of spectrum: Technology, management, and regime change", Journal on Telecommunications \& High Technology Law, 4:123, 2005.

[8] M.M. Halldorsson, J. Y. Halpern, L. Li, and V.S. Mirrokni, "On spectrum sharing games", in ACM Symposium on Principles of Distributed Computing, pages 107-114, 2004.

[9] K. Hinderer, "On the Structure of Solutions of Stochastic Dynamic Programs", in Proc. of the Seventh Conference on Probability Theory, Brasov, Romania, 1982.

[10] Z. Ji and K.J.R Liu, "Collusion-resistant dynamic spectrum allocation for wireless networks via pricing", in Proceedings of DySPAN 2007, pages 187-190, 2007.

[11] J. Kleinberg and E. Tardos, Algorithm Design, Addison Wesley, 2005.

[12] W. Lehr and J. Crowcroft, "Managing shared access to a spectrum commons", in Proceedings of the rst IEEE Symposium on New Frontiers in Dynamic Spectrum Access Networks, 2005.

[13] N. Nie and C. Comaniciu, "Adaptive channel allocation spectrum etiquette for cognitive radio networks", Mobile Networks and Applications, 11(6):779-797, Dec 2006.

[14] J. Peha, "Spectrum management policy options", IEEE Commun. Surv., 1998.

[15] J. M. Peha and S. Panichpapiboon, "Real-time secondary markets for spectrum", Telecommunications Policy, 28(7-8):603-618, 2004.

[16] J. Peha, "Approaches to spectrum sharing", IEEE Commun. Magazine, pages 10-12, 2005.

[17] C. Peng, H. Zheng, and B.Y. Zhao, "Utilization and fairness in spectrum assignment for opportunistic spectrum access", Mobile Networks and Applications, 11(4):555-576, Aug 2006.

[18] M. Puterman, Markov Decision Processes, Wiley, 1994.

[19] S. Ross, Introduction to Stochastic Dynamic Programming, Academic Press, 1983.

[20] A. Sahasrabudhe and K. Kar, "Bandwidth allocation games under budget and access constraints", in Proceedings of CISS, Princeton, NJ, March 2008.

[21] S. Subramani, T. Basar, S. Armour, D. Kaleshi, and Z. Fan, "Noncooperative equilibrium solutions for spectrum access in distributed cognitive radio networks", in Proceedings of DySPAN 2008, pages 1-5, 2008.

[22] A.P Subramanian et al., "Fast spectrum allocation in coordinated dynamic spectrum access based cellular networks", in Proceedings of DYSPAN 2007, pages 320-330, 2007.

[23] T.M. Valletti, "Spectrum trading", Telecommunications Policy, 25:655670, 2001.

[24] D. West, Introduction to Graph Theory, 2nd ed., Prentice Hall, 2000.

\section{APPENDIX}

Notation: Let $R$ denote the set of real numbers.

First, we note that with $X_{i}$ as in Assumption 1, the assumption $X_{i} \leq_{s t} X_{i^{\prime}}$ for $i \leq i^{\prime}$ is equivalent to the following condition [19]:

Condition 1: For every increasing function $f(i)$,

$$
E\left(f\left(X_{i}\right)\right) \leq E\left(f\left(X_{i^{\prime}}\right)\right) \forall i \leq i^{\prime}
$$

i.e., $\sum_{j} Q_{i j} f(j), \sum_{j} H_{i j}^{G} f(j)$ and $\sum_{j} H_{i j}^{O} f(j)$ are increasing functions of $i$.

Note that in the summations in Condition 1, as well as in those in the rest of this section, the summation is over all possible states of the respective Markov Chain. 


\section{A. Proof of Theorem 1}

We first prove that the value function is concave in $a$ (Theorem 7). Then, using Theorem 7, we prove Theorem 1. We start with a simple lemma, which is used in the proof of Theorem 7.

Lemma 2: For fixed $i, c_{G}, c_{O}, V_{n}\left(a, i, c_{G}, c_{O}\right)$ decreases in $a$.

Proof: We prove the result by induction. Let $V_{0}\left(a, i, c_{G}, c_{O}\right)=0$. Then the claim is true for $n=0$. Suppose $V_{n-1}\left(a, i, c_{G}, c_{O}\right)$ decreases in $a$ for each $i, c_{G}, c_{O}$. Now, let $a_{1} \geq 1$ and $x_{n}^{*}\left(a_{1}, i, c_{G}, c_{O}\right)=x_{1}$ for some $x_{1}$. Then, by (5):

$$
V_{n}\left(a_{1}, i, c_{G}, c_{O}\right)=W_{n}\left(a_{1}, i, c_{G}, c_{O}, x_{1}\right)
$$

Now,

$$
\begin{aligned}
& V_{n}\left(a_{1}-1, i, c_{G}, c_{O}\right) \\
\geq & W_{n}\left(a_{1}-1, i, c_{G}, c_{O}, x_{1}\right) \\
= & \alpha(n) c_{G} x_{1}+J\left(x_{1}+a_{1}-1, i, c_{O}\right)+ \\
& \sum_{d_{G}} \sum_{d_{O}} H_{c_{G} d_{G}}^{G} H_{c_{O} d_{O}}^{O} \sum_{j} Q_{i j} V_{n-1}\left(a_{1}+x_{1}-1, j, d_{G}, d_{O}\right) \\
\geq & \alpha(n) c_{G} x_{1}+J\left(x_{1}+a_{1}, i, c_{O}\right)+ \\
& \sum_{d_{G}} \sum_{d_{O}} H_{c_{G} d_{G}}^{G} H_{c_{O} d_{O}} \sum_{j} Q_{i j} V_{n-1}\left(a_{1}+x_{1}, j, d_{G}, d_{O}\right) \\
= & W_{n}\left(a_{1}, i, c_{G}, c_{O}, x_{1}\right) \\
= & V_{n}\left(a_{1}, i, c_{G}, c_{O}\right) \quad \text { is }
\end{aligned}
$$

where the second inequality follows from induction hypothesis and Property 1 . The result follows.

Theorem 7: For each $n, V_{n}\left(a, i, c_{G}, c_{O}\right)$ is concave in $a$ for fixed $i, c_{G}, c_{O}$.

Proof: We prove the result by induction. $V_{0}\left(a, i, c_{G}, c_{O}\right)$ is concave in $a$ since it is equal to 0 . Suppose $V_{n-1}\left(a, i, c_{G}, c_{O}\right)$ is concave in $a$ for fixed $i, c_{G}, c_{O}$. Recall that $V_{n-1}\left(a, i, c_{G}, c_{O}\right)$ is defined for integer values of $a$. Now, for fixed $i, c_{G}$ and $c_{O}$, define $\tilde{V}_{n-1}\left(a, i, c_{G}, c_{O}\right)$ for $a$ real as the function obtained by linearly interpolating $V_{n-1}\left(a, i, c_{G}, c_{O}\right)$ between each pair of adjacent integers $a_{0}$ and $a_{0}+1$. Similarly, define $\tilde{J}\left(a, i, c_{O}\right)$.

Now, $J\left(x+a, i, c_{O}\right)$ (respectively, $V_{n-1}\left(x+a, i, c_{G}, c_{O}\right)$ ) is concave decreasing in $x+a$ for fixed $i, c_{O}$ (respectively, for fixed $i, c_{G}, c_{O}$ ) by Properties 1 and 2 (respectively, by Lemma 2 and induction hypothesis). Hence, we get:

Property 4: $\tilde{J}\left(x+a, i, c_{O}\right)$ (respectively, $\tilde{V}_{n-1}(x+$ $\left.\left.a, i, c_{G}, c_{O}\right)\right)$ is concave decreasing in $x+a$ for fixed $i, c_{O}$ (respectively, for fixed $i, c_{G}, c_{O}$ ).

Now, consider the function

$$
\begin{array}{r}
\tilde{W}_{n}\left(a, i, c_{G}, c_{O}, x\right)=\alpha(n) c_{G} x+\tilde{J}\left(x+a, i, c_{O}\right) \\
+\sum_{d_{G}} \sum_{d_{O}} H_{c_{G} d_{G}}^{G} H_{c_{O} d_{O}}^{O} \sum_{j} Q_{i j} \tilde{V}_{n-1}\left(a+x, j, d_{G}, d_{O}\right)
\end{array}
$$

as a function of the two real variables $a, x$, i.e. the vector $(a, x)$.

Recall the following property of composition of functions [3]:
Property 5: Let $h: R \rightarrow R, g: R^{k} \rightarrow R$, where $k \geq$ 1 and $R^{k}$ denotes the $k$-dimensional Euclidean space. Let $f: R^{k} \rightarrow R$ be defined by $f(\mathbf{v})=h(g(\mathbf{v}))$. If $h($.$) is$ concave and decreasing, and $g(\mathbf{v})$ is convex in $\mathbf{v}$, then $f(\mathbf{v})$ is concave in $\mathbf{v}$.

By the fact that $a+x$ is convex in $(a, x)$, Property 4 and Property 5, it follows that $\tilde{J}\left(x+a, i, c_{O}\right)$ (respectively, $\left.\tilde{V}_{n-1}\left(a+x, j, d_{G}, d_{O}\right)\right)$ is concave in $(a, x)$ for fixed $i, c_{O}$ (respectively, for fixed $j, d_{G}, d_{O}$ ). Also, $x$ is clearly concave in $(a, x)$. Hence, $\tilde{W}_{n}\left(a, i, c_{G}, c_{O}, x\right)$ being a nonnegative weighted linear combination of these functions, is concave in $(a, x)$ for fixed $i, c_{G}, c_{O}$.

Now, define:

$$
\tilde{V}_{n}\left(a, i, c_{G}, c_{O}\right)=\sup _{x \in R, 0 \leq x \leq M-a} \tilde{W}_{n}\left(a, i, c_{G}, c_{O}, x\right)
$$

Note that $\{x: x \in R, 0 \leq x \leq M-a\}$ is a non-empty convex set. Recall the following property [3]:

Property 6: If $f(a, x)$ is concave in $(a, x)$ and $C$ is a convex nonempty set, then the function

$$
g(a)=\sup _{x \in C} f(a, x)
$$

is concave in $a$, provided $g(a)<\infty$ for some $a$.

Now, $\tilde{V}_{n}\left(a, i, c_{G}, c_{O}\right)<\infty$ (assuming that $c_{G}$ and $c_{O}$ are upper bounded). So by Property $6, \tilde{V}_{n}\left(a, i, c_{G}, c_{O}\right)$ is concave in $a$ for fixed $i, c_{G}, c_{O}$.

Now, we will show that $V_{n}\left(a, i, c_{G}, c_{O}\right)=\tilde{V}_{n}\left(a, i, c_{G}, c_{O}\right)$ for $a$ integer, which will imply that $V_{n}\left(a, i, c_{G}, c_{O}\right)$ is concave.

Fix $i, c_{G}, c_{O}$ and an integer $a$. Note that $V_{n}\left(a, i, c_{G}, c_{O}\right)$ is the maximum of $\tilde{W}_{n}\left(a, i, c_{G}, c_{O}, x\right)$ over integer $x$, whereas $\tilde{V}_{n}\left(a, i, c_{G}, c_{O}\right)$ is the supremum over real $x$ in the range $[0, M-a]$. Hence, to prove that $V_{n}\left(a, i, c_{G}, c_{O}\right)=$ $\tilde{V}_{n}\left(a, i, c_{G}, c_{O}\right)$, it will suffice to show that the supremum over real $x$ occurs at integer $x$.

Now, by the definition of the functions $\tilde{J}($.$) and \tilde{V}_{n-1}($.$) ,$ $f(x)=\tilde{W}_{n}\left(a, i, c_{G}, c_{O}, x\right)$ is continuous and piecewise linear in $x$, with breakpoints at the integers. Also, note that the endpoints of the domain of $f(x)$, viz. 0 and $M-a$ are integers that are contained in the domain. As a result, it can be checked that the maximum of $f(x)$ must occur at an integer. This completes the proof.

Note that $W_{n}\left(a, i, c_{G}, c_{O}, x\right)$ is concave in $(a, x)$ and $V_{n}\left(a, i, c_{G}, c_{O}\right)$ is the maximum of $W_{n}($.$) over a non-convex$ set, namely the set of integers in $[0, M-a]$. This makes the above proof more involved, since had the maximum been over a convex set, the concavity of $V_{n}\left(a, i, c_{G}, c_{O}\right)$ would have simply followed from Property 6.

Proof: [Proof of Theorem 1] From (6), we have:

$$
\begin{aligned}
W_{n}\left(a, i, c_{G}, c_{O}, x\right)= & W_{n}\left(a+1, i, c_{G}, c_{O}, x-1\right) \\
& +\alpha(n) c_{G}, \forall x \geq 1
\end{aligned}
$$

Now, by optimality of $x_{n}^{*}\left(a, i, c_{G}, c_{O}\right)$ :

$W_{n}\left(a, i, c_{G}, c_{O}, x_{n}^{*}\left(a, i, c_{G}, c_{O}\right)\right) \geq W_{n}\left(a, i, c_{G}, c_{O}, x\right) \forall x \geq 1$ 
If $x_{n}^{*}\left(a, i, c_{G}, c_{O}\right) \geq 1$, then from (12) and (13) and some algebra, we get:

$$
\begin{array}{r}
W_{n}\left(a+1, i, c_{G}, c_{O}, x_{n}^{*}\left(a, i, c_{G}, c_{O}\right)-1\right) \\
\geq W_{n}\left(a+1, i, c_{G}, c_{O}, x-1\right) \quad \forall x \geq 1
\end{array}
$$

which shows that $x_{n}^{*}\left(a+1, i, c_{G}, c_{O}\right)=x_{n}^{*}\left(a, i, c_{G}, c_{O}\right)-1$ if $x_{n}^{*}\left(a, i, c_{G}, c_{O}\right) \geq 1$.

Now, suppose $x_{n}^{*}\left(a, i, c_{G}, c_{O}\right)=0$. By Theorem 7 and Property 2, since $V_{n-1}\left(a+x, j, d_{G}, d_{O}\right)$ and $J\left(x+a, i, c_{O}\right)$ are concave in $x$ for fixed $a, j, d_{G}, d_{O}, i, c_{O}$, it follows from (6) that $W_{n}\left(a, i, c_{G}, c_{O}, x\right)$ is concave in $x$. For $x \geq 2$, we have:

$$
\begin{aligned}
& W_{n}\left(a+1, i, c_{G}, c_{O}, x-1\right)-W_{n}\left(a+1, i, c_{G}, c_{O}, 0\right) \\
= & W_{n}\left(a, i, c_{G}, c_{O}, x\right)-W_{n}\left(a, i, c_{G}, c_{O}, 1\right)(\text { by }(12)) \\
\leq \quad & W_{n}\left(a, i, c_{G}, c_{O}, x-1\right)-W_{n}\left(a, i, c_{G}, c_{O}, 0\right) \\
& \quad(\text { by concavity }) \\
\leq \quad & 0\left(\text { since } x_{n}^{*}\left(a, i, c_{G}, c_{O}\right)=0\right)
\end{aligned}
$$

which shows that $x_{n}^{*}\left(a+1, i, c_{G}, c_{O}\right)=0$.

\section{B. Proofs of Theorems 2 and 3}

The proofs of Theorems 2 and 3 are based on the concepts of submodularity and supermodularity, which we briefly review. Let $I \subseteq R$ and $X \subseteq R$ be two sets. A function $g(i, x): I \times X \rightarrow R$ is called supermodular [18] if for $i^{+} \geq i^{-}$in $I$ and $x^{+} \geq x^{-}$in $X$,

$$
g\left(i^{+}, x^{+}\right)+g\left(i^{-}, x^{-}\right) \geq g\left(i^{+}, x^{-}\right)+g\left(i^{-}, x^{+}\right)
$$

If the inequality is reversed, $g$ is called submodular [18].

We will require the following key result [18].

Theorem 8: If $g(i, x)$ is supermodular (submodular) on $I \times X$, then the (largest) maximizer of $g(i, x)$ for a given $i$ :

$$
f(i)=\max \left\{x^{\prime}: x^{\prime} \in \underset{x}{\operatorname{argmax}} g(i, x)\right\}
$$

is increasing (decreasing) in $i$.

To prove Theorem 2 , we show that $W_{n}\left(a, i, c_{G}, c_{O}, x\right)$ is submodular in $(i, x)$. The monotonicity of $x_{n}^{*}\left(a, i, c_{G}, c_{O}\right)$ in $i$ then follows from Theorem 8. First, we prove some lemmas.

The following lemma provides a necessary and sufficient condition for submodularity.

Lemma 3: Let $g(i, x)$ be a function with domain being integer values of $x$ and real values of $i . g(i, x)$ is submodular in $(i, x)$ if and only if $g(i, x)-g(i, x+1)$ is an increasing function of $i$ for all $x$.

Proof: The necessity directly follows from the definition of submodularity. We now prove sufficiency. For an integer $z>0$ :

$$
\begin{array}{r}
g(i, x)-g(i, x+z)=[g(i, x)-g(i, x+1)]+\ldots \\
+[g(i, x+z-1)-g(i, x+z)]
\end{array}
$$

So $g(i, x)-g(i, x+z)$, being the sum of increasing functions, is increasing in $i$.
Hence, for $x^{-}<x^{+}, g\left(i, x^{-}\right)-g\left(i, x^{+}\right)$is increasing in $i$. So for $i^{-}<i^{+}$:

$$
g\left(i^{-}, x^{-}\right)-g\left(i^{-}, x^{+}\right) \leq g\left(i^{+}, x^{-}\right)-g\left(i^{+}, x^{+}\right)
$$

Hence, $g(i, x)$ is submodular in $(i, x)$ by definition.

For $m \geq 1$, define ${ }^{4}$

$$
i_{n}^{m}\left(a, c_{G}, c_{O}\right)=\max \left\{i: x_{n}^{*}\left(a, i, c_{G}, c_{O}\right) \geq m\right\} .
$$

Lemma 4: If $x_{n}^{*}\left(a, i, c_{G}, c_{O}\right)$ is monotone decreasing in $i$, then

$$
i_{n}^{1}\left(a, c_{G}, c_{O}\right) \geq i_{n}^{2}\left(a, c_{G}, c_{O}\right) \geq \ldots \geq i_{n}^{M-a}\left(a, c_{G}, c_{O}\right)
$$

Also, $x_{n}^{*}\left(a, i, c_{G}, c_{O}\right)=m$ if and only if $i_{n}^{m}\left(a, c_{G}, c_{O}\right) \geq$ $i>i_{n}^{m+1}\left(a, c_{G}, c_{O}\right)$.

Proof: The result follows by definition of $i_{n}^{m}($.$) .$

The next lemma establishes a sufficient condition for monotonicity of $x_{n}^{*}\left(i, a, c_{G}, c_{O}\right)$.

Lemma 5: Fix $n$. Suppose $V_{n-1}\left(a, j, d_{G}, d_{O}\right)-V_{n-1}(a+$ $\left.1, j, d_{G}, d_{O}\right)$ is an increasing function of $j$ for each $a, d_{G}$ and $d_{O}$. Then $x_{n}^{*}\left(a, i, c_{G}, c_{O}\right)$ is monotone decreasing in $i$ for each $a, c_{G}$ and $c_{O}$.

It is important to note that the lemma requires $V_{n-1}\left(a, j, d_{G}, d_{O}\right)-V_{n-1}\left(a+1, j, d_{G}, d_{O}\right)$ to be increasing in $j$ for a fixed $n$, and asserts that $x_{n}^{*}\left(a, i, c_{G}, c_{O}\right)$ is monotone decreasing in $i$ for that $n$.

Proof: By (6):

$$
\begin{aligned}
& W_{n}\left(a, i, c_{G}, c_{O}, x\right)-W_{n}\left(a, i, c_{G}, c_{O}, x+1\right) \\
= & -\alpha(n) c_{G}+\left[J\left(a+x, i, c_{O}\right)-J\left(a+x+1, i, c_{O}\right)\right] \\
& +\sum_{d_{G}} \sum_{d_{O}} H_{c_{G} d_{G}}^{G} H_{c_{O} d_{O}}^{O} \sum_{j} Q_{i j}\left(V_{n-1}\left(a+x, j, d_{G}, d_{O}\right)\right. \\
& \left.-V_{n-1}\left(a+x+1, j, d_{G}, d_{O}\right)\right)
\end{aligned}
$$

The first term on the right hand side is constant, the second term is increasing in $i$ by Property 3 and the third term is increasing in $i$ since $V_{n-1}\left(a+x, j, d_{G}, d_{O}\right)-V_{n-1}(a+x+$ $\left.1, j, d_{G}, d_{O}\right)$ is increasing in $j$ and by Condition 1 .

So $W_{n}\left(a, i, c_{G}, c_{O}, x\right)-W_{n}\left(a, i, c_{G}, c_{O}, x+1\right)$ is increasing in $i$. Hence, by Lemma $3, W_{n}\left(a, i, c_{G}, c_{O}, x\right)$ is submodular in $(i, x)$ and so by Theorem $8, x_{n}^{*}\left(a, i, c_{G}, c_{O}\right)$ is monotone decreasing in $i$.

The next lemma is a simple consequence of (8).

Lemma 6: Fix $n$. If $x_{n}^{*}\left(a, i, c_{G}, c_{O}\right)$ is monotone decreasing in $i$ for each $a, c_{G}, c_{O}$, then $i_{n}^{m+1}\left(a, c_{G}, c_{O}\right)=i_{n}^{m}(a+$ $\left.1, c_{G}, c_{O}\right)$ for $m=1,2, \ldots$.

Lemma 7: For each $n, V_{n}\left(a, i, c_{G}, c_{O}\right)-V_{n}(a+$ $\left.1, i, c_{G}, c_{O}\right)$ is an increasing function of $i$ for each $a, c_{G}, c_{O}$.

Proof: We prove the claim by induction. Since $V_{0}\left(a, i, c_{G}, c_{O}\right) \equiv 0$, the claim is true for $n=0$.

Suppose the statement is true for $n-1$, i.e., $V_{n-1}\left(a, j, d_{G}, d_{O}\right)-V_{n-1}\left(a+1, j, d_{G}, d_{O}\right)$ is an increasing function of $j$ for each $a, d_{G}, d_{O}$. Then by Lemma 5 , $x_{n}^{*}\left(a, i, c_{G}, c_{O}\right)$ is monotone decreasing in $i$. Hence, by Lemma $6, i_{n}^{m+1}\left(a, c_{G}, c_{O}\right)=i_{n}^{m}\left(a+1, c_{G}, c_{O}\right)$ for $m=$ $1,2, \ldots$.

${ }^{4}$ If $x_{n}^{*}\left(a, i, c_{G}, c_{O}\right)<m \forall i$, then let $i_{n}^{m}\left(a, c_{G}, c_{O}\right)$ be equal to the smallest demand state. 
Now, we show that $V_{n}\left(a, i, c_{G}, c_{O}\right)-V_{n}\left(a+1, i, c_{G}, c_{O}\right)$ is an increasing function of $i$. Fix $a, c_{G}$ and $c_{O}$. We have the following cases:

Case 1: $i>i_{n}^{1}\left(a, c_{G}, c_{O}\right)$

By Lemma 4 and Lemma 6:

$$
i>i_{n}^{1}\left(a, c_{G}, c_{O}\right) \geq i_{n}^{2}\left(a, c_{G}, c_{O}\right)=i_{n}^{1}\left(a+1, c_{G}, c_{O}\right)
$$

So by Lemma $4, x_{n}^{*}\left(a, i, c_{G}, c_{O}\right)=x_{n}^{*}\left(a+1, i, c_{G}, c_{O}\right)=0$. Hence, by (5) and (6):

$$
\begin{aligned}
& V_{n}\left(a, i, c_{G}, c_{O}\right)-V_{n}\left(a+1, i, c_{G}, c_{O}\right) \\
= & W_{n}\left(a, i, c_{G}, c_{O}, 0\right)-W_{n}\left(a+1, i, c_{G}, c_{O}, 0\right) \\
= & \left(J\left(a, i, c_{O}\right)-J\left(a+1, i, c_{O}\right)\right) \\
& +\sum_{d_{G}} \sum_{d_{O}} H_{c_{G} d_{G}}^{G} H_{c_{O} d_{O}}^{O} \sum_{j} Q_{i j}\left(V_{n-1}\left(a, j, d_{G}, d_{O}\right)\right. \\
& \left.-V_{n-1}\left(a+1, j, d_{G}, d_{O}\right)\right)
\end{aligned}
$$
1.

Case 2: $i_{n}^{m}\left(a, c_{G}, c_{O}\right) \geq i>i_{n}^{m+1}\left(a, c_{G}, c_{O}\right)$, where $m \geq$ By Lemma $4, x_{n}^{*}\left(a, i, c_{G}, c_{O}\right)=m$ and hence by Theorem 1 , $x_{n}^{*}\left(a+1, i, c_{G}, c_{O}\right)=m-1$. So by (5) and (6) and some cancellation of terms, we get:

$$
\begin{aligned}
& V_{n}\left(a, i, c_{G}, c_{O}\right)-V_{n}\left(a+1, i, c_{G}, c_{O}\right) \\
= & W_{n}\left(a, i, c_{G}, c_{O}, m\right)-W_{n}\left(a+1, i, c_{G}, c_{O}, m-1\right) \\
= & \alpha(n) c_{G}
\end{aligned}
$$

By (15) and (16), $V_{n}\left(a, i, c_{G}, c_{O}\right)-V_{n}\left(a+1, i, c_{G}, c_{O}\right)$

$=\left\{\begin{array}{lr}\alpha(n) c_{G} & \text { if } i \leq i_{n}^{1}\left(a, c_{G}, c_{O}\right), \\ \left(J\left(a, i, c_{O}\right)-J\left(a+1, i, c_{O}\right)\right) & \\ +\sum_{d_{G}} \sum_{d_{O}} H_{c_{G}}^{G} d_{G} H_{c_{O} d_{O}}^{O} \sum_{j} Q_{i j}\left(V_{n-1}\left(a, j, d_{G}, d_{O}\right)\right. \\ \left.-V_{n-1}\left(a+1, j, d_{G}, d_{O}\right)\right) & \text { if } i>i_{n}^{1}\left(a, c_{G}, c_{O}\right) .\end{array}\right.$

The expression for $V_{n}\left(a, i, c_{G}, c_{O}\right)-V_{n}\left(a+1, i, c_{G}, c_{O}\right)$ for $i>i_{n}^{1}\left(a, c_{G}, c_{O}\right)$ is an increasing function of $i$ by Property 3 , induction hypothesis and Condition 1. Thus, to show that $V_{n}\left(a, i, c_{G}, c_{O}\right)-V_{n}\left(a+1, i, c_{G}, c_{O}\right)$ is increasing in $i$, it is sufficient to show that for $i>i_{n}^{1}\left(a, c_{G}, c_{O}\right)$ :

$$
\begin{aligned}
&\left(J\left(a, i, c_{O}\right)-J\left(a+1, i, c_{O}\right)\right) \\
&+ \sum_{d_{G}} \sum_{d_{O}} H_{c_{G} d_{G}}^{G} H_{c_{O} d_{O}}^{O} \sum_{j} Q_{i j}\left(V_{n-1}\left(a, j, d_{G}, d_{O}\right)\right. \\
&\left.-\quad V_{n-1}\left(a+1, j, d_{G}, d_{O}\right)\right) \geq \alpha(n) c_{G}
\end{aligned}
$$

By (6), (17) is equivalent to $W_{n}\left(a, i, c_{G}, c_{O}, 0\right) \geq$ $W_{n}\left(a, i, c_{G}, c_{O}, 1\right)$, which is true because $x_{n}^{*}\left(a, i, c_{G}, c_{O}\right)=$ 0 for $i>i_{n}^{1}\left(a, c_{G}, c_{O}\right)$. The result follows.

From the above lemmas, we get the desired monotonicity of $x_{n}^{*}\left(i, a, c_{G}, c_{O}\right)$.

Proof: [Proof of Theorem 2] Fix $n, a, c_{G}$ and $c_{O}$. By Lemma $7, V_{n-1}\left(a, j, d_{G}, d_{O}\right)-V_{n-1}\left(a+1, j, d_{G}, d_{O}\right)$ is an increasing function of $j$ for each $d_{G}, d_{O}$. The result follows by Lemma 5.

Proof: [Proof of Theorem 3] The proof is very similar to the proof of Theorem 2 and hence omitted.

\section{Proofs of results in Section III}

Proof: [Proof of Theorem 4] We show that the Maximum Independent Set (MIS) problem is a special case of Primary-SCTM. Consider the following special case of Primary-SCTM: $M=1, T=1$. At each node, the primary's demand is always 0 , and the prices of Type $G$ and $O$ contracts are fixed, equal to $\frac{1}{2}$ and 1 respectively. Thus, it is optimal never to sell a type $G$ contract.

The Primary-SCTM problem reduces to that of finding a maximum independent set of nodes (at which to sell Type $O$ contracts). The result follows, since the MIS problem is NP-Hard [11].

Proof: [Proof of Lemma 1] Let $N_{e, j}^{t}$ be the number of Type- $j$ contracts $(j \in\{G, O\})$ sold by a policy $P$ in slot $t$ on channel $e$. We make the following key observations:

(1) The revenue of any policy depends only on the number of Type- $G$ and Type- $O$ contracts it sells on each channel, in each slot, independent of which nodes it sells them at. That is, the revenue of the policy $P$ is completely determined by:

$$
\left\{N_{e, G}^{t}, N_{e, O}^{t}: e=1, \ldots, M ; t=1, \ldots, T\right\}
$$

This follows from the fact that on each channel, the prices of both types of contracts and the usage status (i.e., whether or not the primary is using the channel for subscriber demand satisfaction) are the same at all nodes.

(2) For every policy, on each channel, at any time, the total number of Type- $G$ and Type- $O$ contracts currently leased is at most equal to $|I(S)|$.

That is, for the above policy $P$, for every slot $t$ :

$$
\sum_{\tau=1}^{t} N_{e, G}^{\tau}+N_{e, O}^{t} \leq|I(S)|, e=1, \ldots, M
$$

This follows from the fact that $I(S)$ is a maximum independent set.

Now, let $P$ be an optimal policy. Consider a policy $f \in \mathcal{F}$, which initially finds a maximum independent set $I(S)$. Also, whenever $P$ sells a contract, $f$ sells the same type of contract on the same channel at a node in $I(S)$ at which no contract has been sold on this channel. More precisely, number the nodes in $I(S)$ from 1 to $|I(S)|$. In slot $t$, on channel $e$, policy $f$ sells Type- $G$ contracts at the nodes $\sum_{\tau=1}^{t-1} N_{e, G}^{\tau}+1$ to $\sum_{\tau=1}^{t} N_{e, G}^{\tau}$ and Type- $O$ contracts at the nodes $\sum_{\tau=1}^{t} N_{e, G}^{\tau}+$ 1 to $\sum_{\tau=1}^{t} N_{e, G}^{\tau}+N_{e, O}^{t}$. It can be checked that on each channel $e$, (a) for policy $f$, two or more contracts never stand leased at the same node and (b) by (18), in each slot $t, f$ finds enough nodes in $I(S)$ to sell contracts at.

Now, by observation (1), the revenue of $f$ is the same as that of $P$, and therefore $f$ is optimal. 